\title{
SMOOTHNESS OF CERTAIN DEGENERATE ELLIPTIC EQUATIONS
}

\author{
JOHN L. LEWIS
}

\begin{abstract}
Given $p>1, p \neq 2$, let $u$ be a solution to $\operatorname{div}\left(|\operatorname{grad} u|^{P-2} \operatorname{grad} u\right)=0$ on a domain $D$ in Euclidean two space. We prove that if $u$ is nonconstant and real analytic in $D$, then the gradient of $u$ does not vanish in $D$. Some examples of Krol' are used to show this result and a related result of Ural'tseva are nearly best possible.
\end{abstract}

1. Let $x=\left(x_{1}, x_{2}, \ldots, x_{n}\right)$ denote a point in $n$ dimensional Euclidean space $\mathbf{R}^{n}$ and let $|x|$ denote the norm of $x$. For $x=\left(x_{1}, \ldots, x_{n}\right)$ and $y=\left(y_{1}, \ldots, y_{n}\right)$ in $\mathbf{R}^{n}$, we put $x \cdot y=\sum_{i=1}^{n} x_{i} y_{i}$. Let $D$ be a domain in $\mathbf{R}^{n}$. For fixed $p>1$, let $u$ be a real-valued function on $D$ with distributional derivatives which are locally integrable to the $p$ th power. Let $\nabla u=\left(u_{x_{1}}, \ldots, u_{x_{n}}\right)$ and suppose that

$$
\int_{D}|\nabla u|^{P-2} \nabla u \cdot \nabla \phi d x=0
$$

whenever $\phi$ is infinitely differentiable with compact support in $D$. Here, $|\nabla u|^{P-2} \nabla u$ is defined to be zero at each $x$ where $\nabla u=0$. Observe that if $u$ has continuous second partials in $D$, then (1.1) and the divergence theorem imply for each $x \in D$ that

$$
L u=(p-2) \sum_{k, j=1}^{n} u_{x_{k}} u_{x_{j}} u_{x_{k} x_{j}}+\Delta u|\nabla u|^{2}=0,
$$

where $\Delta u$ denotes the Laplacian of $u$. Therefore, we shall call $u$ a weak solution to (1.2). We remark that the smoothness of $u$ in (1.1) cannot be deduced directly from theorems on weak solutions of elliptic equations of divergence type, since (1.2) is degenerate. The best result appears to be due to Ural'tseva [4], who showed that $u$ has Hölder continuous derivatives in $D$ when $p>2$ (see also Uhlenbeck [3] for the case $p=4$ ). However, if ess inf $|\nabla u|>0$, for each compact subset $K \subseteq D$, then it is well known (see for example [2, p. 208]) that $u$ is real analytic in $D$. The converse of this statement is also true when $D \subseteq \mathbf{R}^{2}$ and $p \neq 2$. We prove

TheOREM 1. Given $p>1, p \neq 2$, let $u$ be a nonconstant real analytic solution to (1.2) in a domain $D \subseteq \mathbf{R}^{2}$. Then $\nabla u$ does not vanish in $D$.

We note that Theorem 1 is false when $p=2$. Consider, for example, $u\left(x_{1}, x_{2}\right)=$ $x_{1} x_{2}$. The proof of Theorem 1 is by contradiction. In Lemma 1 (\$2) we show it

Received by the editors September 24, 1979.

1980 Mathematics Subject Classification. Primary 35B65, $35 \mathrm{~J} 70$.

Key words and phrases. Real analyticity, degenerate elliptic equations of divergence type. 
suffices to prove Theorem 1 when $D=\mathbf{R}^{2}$ and $u$ is a homogeneous polynomial of degree $m \geqslant 2$. Using Lemma 1 , we show in Lemma 2 that $u$ is of the form,

$$
u\left(x_{1}, x_{2}\right)=c\left(x_{1} \pm i x_{2}\right)^{m}, \quad\left(x_{1}, x_{2}\right) \in \mathbf{R}^{2},
$$

where $c$ is a complex constant. Since $u$ is real valued, we then obtain a contradiction to our assumption that Theorem 1 is false. In $\$ 3$ we point out that some examples of Krol' imply Theorem 1 is nearly best possible in the following sense. For each positive integer $k$ and $p>1$, there exists a nonconstant solution $u$ to (1.2) in $\mathbf{R}^{2}$ with continuous derivatives of order $k$ and $\nabla u(0)=0$. These examples also show that a weak solution to (1.2) in $\mathbf{R}^{n}$ need not have continuous second partials when $p>2$. Thus, Ural'tseva's result cannot be improved. Finally in $\$ 4$ we make some remarks concerning possible analogues of Theorem 1 and Lemma 2 in $\mathbf{R}^{n}$, $n>2$.

2. Proof of Theorem 1. Let $u$ be as in Theorem 1 and suppose that $\nabla u$ vanishes somewhere in $D$. Since (1.2) is translation invariant, we may assume that $0 \in D$ and $\nabla u(0)=0$. Under these assumptions, we prove

LEMMA 1. There exists a real-valued homogeneous polynomial $Q$ on $\mathbf{R}^{2}$ with degree $m \geqslant 2$ and $L Q \equiv 0$.

Proof. Since $u$ is a nonconstant real analytic function on $D$ and $\nabla u(0)=0$, there exists $\rho>0$ and a positive integer $m \geqslant 2$, such that $u(x)-u(0)=Q(x)+$ $g(x),|x|<\rho$, where $Q$ is a real-valued homogeneous polynomial of degree $m$ and $g$ is real analytic in $|x|<\rho$ with $g(x)=O\left(|x|^{m+1}\right)$ as $x \rightarrow 0$. Hence

$$
\begin{aligned}
u_{x_{i}} & =Q_{x_{i}}+O\left(|x|^{m}\right), \quad i=1,2, \\
u_{x_{i} x_{j}} & =Q_{x_{i} x_{j}}+O\left(|x|^{m-1}\right), \quad i, j=1,2 .
\end{aligned}
$$

Substituting these equalities into (1.2) it follows that

$$
0=L u\left(x_{1}, x_{2}\right)=L Q\left(x_{1}, x_{2}\right)+O\left(|x|^{3 m-3}\right), \quad|x|<\rho .
$$

We observe that $L Q$ is a homogeneous polynomial of degree $3 m-4$. If $|x|=r$, $x_{1}=r \cos \theta$ and $x_{2}=r \sin \theta, 0 \leqslant \theta<2 \pi$, then, from this observation and (2.1), we get

$$
\begin{aligned}
0 & =\lim _{r \rightarrow 0} r^{4-3 m} L u(r \cos \theta, r \sin \theta)=\lim _{r \rightarrow 0} r^{4-3 m} L Q(r \cos \theta, r \sin \theta) \\
& =L Q(\cos \theta, \sin \theta) .
\end{aligned}
$$

This equality and the homogeneity of $L Q$ imply Lemma 1 .

Next we prove

LemMa 2. Let $Q$ be a homogeneous polynomial on $\mathbf{R}^{2}$ with complex coefficients and degree $m \geqslant 2$. Given $p>1, p \neq 2$, suppose that $L Q \equiv 0$. Then $Q$ is of the form

$$
Q\left(x_{1}, x_{2}\right)=c\left(x_{1} \pm i x_{2}\right)^{m}, \quad\left(x_{1}, x_{2}\right) \in \mathbf{R}^{2}
$$

where $c$ is a complex constant.

Proof. Let $\mathrm{C}$ denote the complex numbers. Observe that $Q$ has an extension to $\mathrm{C}^{2}$, which is analytic in $x_{1}, x_{2}$, separately. Let the partial derivatives of $Q$ with 
respect to $x_{1}, x_{2} \in \mathrm{C}$ be denoted in the usual way. Then from the identity theorem for analytic functions, it follows that $L Q \equiv 0$ on $\mathbf{C}^{2}$. Throughout the proof of Lemma 2, we shall use the following remark. If $P$ is a homogeneous polynomial of degree $k$ on $\mathbf{C}^{2}$ and $P\left(y_{1}, y_{2}\right)=0$ for some $\left(y_{1}, y_{2}\right) \in \mathbf{C}^{2}-\{0\}$, then $-x_{2} y_{1}+x_{1} y_{2}$ divides $P$. Hence, $P$ can be written as a product of $k$ linear factors in $x_{1}$ and $x_{2}$. It follows from this representation of $P$ that if $P_{x_{1}} r\left(y_{1}, y_{2}\right)=0$ for $r, s$ integers and $0 \leqslant r \leqslant s$, then $\left(-x_{2} y_{1}+x_{1} y_{2}\right)^{s+1}$ divides $P$.

We first show

There exists $\left(y_{1}, y_{2}\right) \in \mathbf{C}^{2}-\{0\}$, such that $Q_{x_{1}}\left(y_{1}, y_{2}\right)=0=$ $Q_{x_{2}}\left(y_{1}, y_{2}\right)$.

To prove (2.2), suppose $\left(-x_{2} y_{1}+x_{1} y_{2}\right)^{s+1}$ divides $Q_{x_{1}}$ for some nonnegative integer $s$ and $\left(y_{1}, y_{2}\right) \in \mathbf{C}^{2}-\{0\}$. Then $Q_{x_{1}}\left(y_{1}, y_{2}\right)=0$, and

$$
Q_{x_{1}^{\prime+1}}\left(y_{1}, y_{2}\right)=Q_{x_{2} x_{1}^{r}}\left(y_{1}, y_{2}\right)=0 \text {, }
$$

when $s \geqslant 1$ and $1 \leqslant r \leqslant s$. Using the equality, we obtain

$$
0=(L Q)_{x_{1}^{r}}\left(y_{1}, y_{2}\right)=\left[\left(Q_{x_{2}}\right)^{2}\left(Q_{x_{1}^{r+2}}+(p-1) Q_{x_{1}^{r} x_{2}^{2}}\right)\right]\left(y_{1}, y_{2}\right),
$$

for $0 \leqslant r \leqslant s$. Let $R=Q_{x_{1}^{2}}+(p-1) Q_{x_{2}^{2}}$. If $Q_{x_{2}}\left(y_{1}, y_{2}\right) \neq 0$, then from (2.3) and our earlier remark, we see that $\left(-x_{2} y_{1}+x_{1} y_{2}\right)^{s+1}$ divides $R$. Hence, if (2.2) is false, then either $Q_{x_{1}} \equiv 0 \equiv R$ or $Q_{x_{1}}$ divides $R$. In either case

$$
R \equiv 0 \text {, }
$$

since otherwise $Q_{x_{1}}$ is homogeneous of degree $m-1$ and $R$ is homogeneous of degree $m-2$.

If (2.2) is false, we may also interchange the roles of $x_{1}$ and $x_{2}$ to get

$$
(p-1) Q_{x_{1}^{2}}+Q_{x_{2}^{2}} \equiv 0 .
$$

Since $p>1$ and $p \neq 2$, we conclude from (2.4) and (2.5) that $Q_{x_{1}^{2}} \equiv 0 \equiv Q_{x_{2}^{2}}$. From Taylor's theorem, it follows that $m=2$ and $Q\left(x_{1}, x_{2}\right)=A x_{1} x_{2}$, where $A \neq 0$ is a complex constant. However, in this case

$$
L Q=(p-2) A^{3} x_{1} x_{2} \neq 0 .
$$

We have reached a contradiction. Thus, (2.2) is valid.

Next we make some detailed calculations. To simplify our notation we put $z_{2}=-y_{1}, z_{1}=y_{2}$ and

$$
\sigma\left(x_{1}, x_{2}\right)=x_{1} z_{1}+x_{2} z_{2}, \quad x_{1}, x_{2} \in \mathbf{C},
$$

where $y_{1}, y_{2}$ are as in (2.2). We note from (2.2) and Euler's formula for homogeneous functions that $Q\left(-z_{2}, z_{1}\right)=0$. From this equality, (2.2), and the remark following Lemma 2, we see that $Q$ may be written in the form

$$
Q\left(x_{1}, x_{2}\right)=\left(\sigma^{k} T\right)\left(x_{1}, x_{2}\right), \quad\left(x_{1}, x_{2}\right) \in \mathbf{C}^{2},
$$

where $k \geqslant 2$ is an integer and $T\left(-z_{2}, z_{1}\right) \neq 0$. Now for $r, s=1,2$, 


$$
\begin{aligned}
Q_{x_{r}} Q_{x_{s}} & =\left(k z_{r} T+\sigma T_{x_{r}}\right)\left(k z_{s} T+\sigma T_{x_{s}}\right) \sigma^{2 k-2} \\
& =\left[k^{2} z_{r} z_{s} T^{2}+k \sigma T\left(z_{s} T_{x_{r}}+z_{r} T_{x_{s}}\right)+\sigma^{2} T_{x_{r}} T_{x_{s}}\right] \sigma^{2 k-2},
\end{aligned}
$$

and

$$
Q_{x_{r} x_{s}}=\left[k(k-1) z_{r} z_{s} T+k \sigma\left(z_{r} T_{x_{s}}+z_{s} T_{x_{r}}\right)+\sigma^{2} T_{x_{r} x_{s}}\right] \sigma^{k-2}
$$

Multiplying these expressions together, we get

$$
\sigma^{4-3 k} Q_{x_{r}} Q_{x_{s}} Q_{x_{r} x_{s}}=a_{r s}+b_{r s} \sigma+c_{r s} \sigma^{2}+O\left(|\sigma|^{3}\right)
$$

as $\left(x_{1}, x_{2}\right) \rightarrow\left(-z_{2}, z_{1}\right)$, where

$$
\begin{aligned}
& a_{r s}=k^{3}(k-1) z_{r}^{2} z_{s}^{2} T^{3} \\
& b_{r s}=k^{2}(2 k-1) z_{r} z_{s} T^{2}\left(z_{s} T_{x_{r}}+z_{r} T_{x_{s}}\right) \\
& c_{r s}=k(k-1) z_{r} z_{s} T_{x_{r}} T_{x_{s}} T+k^{2} T\left(z_{s} T_{x_{r}}+z_{r} T_{x_{s}}\right)^{2}+k^{2} z_{r} z_{s} T^{2} T_{x_{r} x_{s}}
\end{aligned}
$$

Also

$$
\sigma^{4-3 k}\left(Q_{x_{s}}\right)^{2} Q_{x_{r} x_{r}}=d_{r s}+e_{r s} \sigma+f_{r s} \sigma^{2}+O\left(|\sigma|^{3}\right)
$$

as $\left(x_{1}, x_{2}\right) \rightarrow\left(-z_{2}, z_{1}\right)$, where

$$
\begin{aligned}
& d_{r s}=k^{3}(k-1) z_{r}^{2} z_{s}^{2} T^{3}, \quad e_{r s}=2 k^{3} z_{s}^{2} z_{r} T^{2} T_{x_{r}}+2 k^{2}(k-1) z_{r}^{2} z_{s} T^{2} T_{x_{s}}, \\
& f_{r s}=k(k-1) z_{r}^{2} T\left(T_{x_{s}}\right)^{2}+k^{2} z_{s}^{2} T^{2} T_{x_{r} x_{r}}+4 k^{2} z_{r} z_{s} T_{x_{r}} T_{x_{s}} T .
\end{aligned}
$$

Using (2.7) and (2.8), we obtain

$$
\begin{aligned}
0= & \sigma^{4-3 k} L Q=\sum_{r, s=1}^{2}\left[(p-2) a_{r s}+d_{r s}\right]+\sum_{r, s=1}^{2}\left[(p-2) b_{r s}+e_{r s}\right] \sigma \\
& +\sum_{r, s=1}^{2}\left[(p-2) c_{r s}+f_{r s}\right] \sigma^{2}+O\left(|\sigma|^{3}\right) \\
= & \lambda_{1}+\lambda_{2} \sigma+\lambda_{3} \sigma^{2}+O\left(|\sigma|^{3}\right)
\end{aligned}
$$

as $\left(x_{1}, x_{2}\right) \rightarrow\left(-z_{2}, z_{1}\right)$. Evaluating (2.9) at $\left(-z_{2}, z_{1}\right)$, it follows that

$$
\begin{aligned}
0 & =\lambda_{1}\left(-z_{2}, z_{1}\right)=(p-1) k^{3}(k-1) T^{3}\left(-z_{2}, z_{1}\right) \sum_{r, s=1}^{2} z_{r}^{2} z_{s}^{2} \\
& =(p-1) k^{3}(k-1) T^{3}\left(-z_{2}, z_{1}\right)\left(z_{1}^{2}+z_{2}^{2}\right)^{2} .
\end{aligned}
$$

Thus

$$
z_{1}^{2}+z_{2}^{2}=0
$$

and $\lambda_{1} \equiv 0$. Since each term in $b_{r s}$ or $e_{r s}$ contains one of $z_{r}^{2}, z_{s}^{2}$, we see, from (2.10), that $\lambda_{2} \equiv 0$. Again, from (2.10) and Euler's formula for homogeneous functions, we 
find that

$$
\begin{aligned}
&\left(\sum_{r, s=1}^{2} c_{r s}\right)\left(z_{1}, z_{2}\right) \\
&=\left[T \sum_{r, s=1}^{2} k(k-1) z_{r} z_{s} T_{x_{r}} T_{x_{s}}+k^{2} T \sum_{r, s=1}^{2}\left(z_{s} T_{x_{r}}+z_{r} T_{x_{s}}\right)^{2}\right]\left(z_{1}, z_{2}\right) \\
&+\left[k^{2} T^{2} \sum_{r, s=1}^{2} z_{r} z_{s} T_{x_{r} x_{s}}\right]\left(z_{1}, z_{2}\right) \\
&= {\left[\left(3 k^{2}-k\right) T\left(\sum_{r=1}^{2} z_{r} T_{x_{r}}\right)^{2}\right]\left(z_{1}, z_{2}\right) } \\
&+\left[k^{2}(m-k-1) T^{2}\left(\sum z_{r} T_{x_{r}}\right)\right]\left(z_{1}, z_{2}\right) \\
&= {\left[\left(3 k^{2}-k\right)(m-k)^{2}+k^{2}(m-k)(m-k-1)\right] T^{3}\left(z_{1}, z_{2}\right) . }
\end{aligned}
$$

Similarly,

$$
\left(\sum_{r, s=1}^{2} f_{r s}\right)\left(z_{1}, z_{2}\right)=4 k^{2}(m-k)^{2} T^{3}\left(z_{1}, z_{2}\right) \text {. }
$$

Dividing (2.9) by $\sigma^{2}$ and letting $\left(x_{1}, x_{2}\right) \rightarrow\left(z_{1}, z_{2}\right)$, we deduce from the above equalities that

$$
\begin{aligned}
0 & =\lambda_{3}\left(z_{1}, z_{2}\right) \\
& =\left\{(p-2)\left[\left(4 k^{2}-k\right)(m-k)^{2}-k^{2}(m-k)\right]+4 k^{2}(m-k)^{2}\right\} T^{3}\left(z_{1}, z_{2}\right) .
\end{aligned}
$$

Observe from (2.10) that

$$
\begin{aligned}
0 & \neq T\left(-z_{2}, z_{1}\right)=\left(-z_{2}\right)^{m-k} T\left(1,-z_{1} / z_{2}\right)=\left(-z_{2}\right)^{m-k} T\left(1, z_{2} / z_{1}\right) \\
& =\left(-z_{2} / z_{1}\right)^{m-k} T\left(z_{1}, z_{2}\right) .
\end{aligned}
$$

Hence

$$
h(p)=(p-2)\left[\left(4 k^{2}-k\right)(m-k)^{2}-k^{2}(m-k)\right]+4 k^{2}(m-k)^{2}=0 .
$$

Since, clearly, $d h / d p \geqslant 0$ and

$$
h(1)=k(m-k)^{2}+k^{2}(m-k)>0,
$$

the above equality can hold for $p>1$ only if $m=k$. Thus $T$ is a constant function. From (2.6) and (2.10) we conclude that Lemma 2 is true.

To prove Theorem 1, observe that Lemmas 1 and 2 contradict each other. From this contradiction, we obtain Theorem 1.

3. Some examples. Let $r=|x|, x_{1}=r \cos \theta$ and $x_{2}=r \sin \theta, 0<|\theta|<\pi$, be polar coordinates in $\mathbf{R}^{2}$. For fixed $p>1$ and $\alpha, 0<\alpha<\pi / 2$, we seek positive solutions to (1.2) in $\{x:|\theta|<\alpha\}$ of the form

$$
u(r, \theta)=r^{\lambda} f(|\theta|), \quad|\theta|<\alpha,
$$

where $f$ is infinitely differentiable for $0<\theta<\alpha$, and satisfies the boundary conditions, $f(0)=1, f(\alpha)=f^{\prime}(0)=0$. Following Krol' [1, p. 135] we note that if 
$g(\theta)=f^{\prime}(\theta) / f(\theta), 0 \leqslant \theta<\alpha$, then (1.2) implies that

$$
\left\{\lambda\left(\lambda^{2}+g^{2}\right)^{-1}-(\lambda-1)\left[\lambda^{2}+g^{2}+\lambda(2-p) /(p-1)\right]^{-1}\right\} g^{\prime}(\theta)=-1,
$$

for $0 \leqslant \theta<\alpha$. Let

$$
h(\lambda)=(\lambda-1)\left[\lambda^{2}-\lambda(p-2) /(p-1)\right]^{-1 / 2} .
$$

Integrating (3.2) from 0 to $\alpha$, we find that

$$
h(\lambda)=1-2 \alpha / \pi \text {. }
$$

Now for $\lambda>1$,

$$
h^{\prime}(\lambda) / h(\lambda)=(\lambda-1)^{-1}-(2 \lambda)^{-1}-\{2[\lambda-(p-2) /(p-1)]\}^{-1}>0
$$

since the function $t \rightarrow(\lambda-t)^{-1}$ is increasing for $t<\lambda$. Thus, $h$ is increasing on $(1, \infty)$. Since $h(1)=0$, and $\lim _{\lambda \rightarrow+\infty} h(\lambda)=1$, it follows that (3.3) has a unique solution $\lambda=\lambda(p, \alpha)$ satisfying $\lambda \geqslant 1$ whenever $0<\alpha<\pi / 2$.

For this value of $\lambda$ it is easily verified that the term in brackets in (3.2) is positive. Integrating (3.2), using this fact and the inverse function theorem, we deduce first the existence of $g$ and then the existence of $u$ in (3.1).

If $\alpha=\pi / 2 s, s$ a positive integer, then $u$ extends to a weak solution of (1.2) on $\mathbf{R}^{2}$ by reflection. More specifically, let

$$
u(r, \theta)=(-1)^{j} r^{\lambda} f(|\theta-j \pi / s|), \quad(2 j-1) \pi / 2 s<|\theta|<(2 j+1) \pi / 2 s,
$$

for $0<j \leqslant s$. Clearly, if $\lambda>k, k$ a positive integer, then $u$ has continuous derivatives of order $k$ in $R^{2}$. Moreover, from (3.3) and (3.4) we see for $p$ fixed that $\lambda \rightarrow \infty$ as $\alpha \rightarrow 0$. Hence, if $s$ is large enough, then $u$ in (3.5) has continuous derivatives of order $k$ in $\mathbf{R}^{2}$.

Next, let $s=2$ in (3.5). Then (3.3) defines $\lambda$ implicitly as a continuously differentiable function of $p$ for $1<p<\infty$. Taking logarithmic derivatives in (3.3) and using (3.4), we obtain

$$
2(d \lambda / d p)=-(p-1)^{-2}[\lambda-(p-2) /(p-1)]^{-1}\left[h(\lambda) / h^{\prime}(\lambda)\right]<0,
$$

for $1<p<\infty$. Thus $\lambda$ is decreasing as a function of $p$ on $(1, \infty)$. Since $\lambda(2)=2$, we conclude that $1<\lambda(p)<2, p>2$, and thereupon that $u$ in (3.5) does not have continuous second derivatives in $R^{2}$. Finally, we note from (3.3) that $\lambda \rightarrow 4 / 3$ as $p \rightarrow \infty$.

4. Remark. It appears likely that Theorem 1 remains true when $D \subseteq \mathbf{R}^{n}, n>2$. The proof of Lemma 1 is unchanged. The analogue of Lemma 2 should be that a homogeneous polynomial $Q$ with complex coefficients and degree $m>2$ is a solution to (1.2) if and only if

$$
H^{3} \equiv 0
$$

where $H=\left(Q_{x_{i} x_{j}}\right)$ is the Hessian matrix of $Q$. To see why, note from Euler's formula for homogeneous functions, that (1.2) may be written in matrix form as

$$
X\left[(p-2) H^{3}+(\operatorname{tr} H) H^{2}\right] X^{T} \equiv 0,
$$


where $X=\left(x_{1}, \ldots, x_{n}\right), X^{T}$ is the transpose of $X$ and $\operatorname{tr} H$ denotes the trace of $H$. Clearly, (4.1) implies (4.2). Also, if $Q$ satisfies (4.1), then $Q$ does not have real coefficients, since all the characteristic roots of $H$ are zero. If $m=2$ and $Q$ satisfies (4.2), then

$$
(p-2) H^{3}+(\operatorname{tr} H) H^{2}=0,
$$

since in this case $H$ is a constant matrix. It follows from this equation that if $\mu$ is a characteristic root of $H$, then either $\mu=0$ or $\mu=-(p-2)^{-1}(\operatorname{tr} H)$. Summing the characteristic roots of $H$, we deduce that

$$
\operatorname{tr} H=-k(p-2)^{-1}(\operatorname{tr} H)
$$

for some nonnegative integer $k$. Since $p-2+k \neq 0$ when $p>1, p \neq 2$, we conclude that tr $H=0$. Hence (4.2) implies (4.1) when $m=2$.

It seems quite difficult to show (4.2) implies (4.1) for $m>2$. Here the method used in $\mathbf{R}^{2}$ fails completely and the coefficient relations obtained from (4.2) are rather complicated. However, using these relations, the author has succeeded in showing there are no real homogeneous polynomials of degree three in $\mathbf{R}^{3}$ which satisfy (4.2). We omit the details.

\section{REFERENCES}

1. I. Krol', On the behavior of the solutions of a quasilinear equation near null salient points of the boundary, Proc. Steklov Inst. Math. 125 (1973), 130-136.

2. J. Lewis, Capacitary functions in convex rings, Arch. Rational Mech. Anal. 66 (1977), 201-224.

3. K. Uhlenbeck, Regularity for a class of nonlinear elliptic systems, Acta Math. 138 (1977), 219-240.

4. N. Ural'tseva, Degenerate quasilinear elliptic systems, Zap. Naučn. Sem. Leningrad. Otdel. Mat. Inst. Steklov 7 (1968), 184-222.

Departiment of Mathematics, University of Kentucky, LeXington, Kentucky 40506 